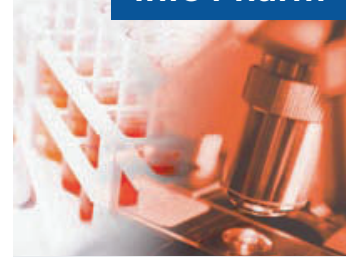

Berichte aus der Pharmaindustrie

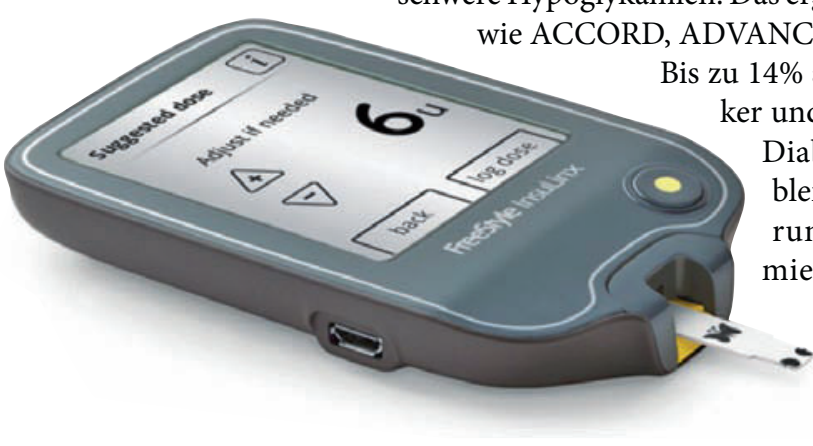
Diabetiker haben Probleme mit Unterzuckerungen. „Hypoglykämien sind ein starker Prädiktor für kardiovaskulären Tod“, so Kröger.
Möglicherweise erhöhen sie auch das Risiko für Demenz, wie eine Längsschnittkohortenstudie mit fast 17000 Patienten nahelegt [1]. Die Angst vor Hypoglykämien reduziert die Compliance der Patienten, die sich ohnehin mit dem Selbstmanagement ihrer Erkrankung überfordert fühlen können, wie Kröger sagte. Sie haben häufig Probleme, die Therapie in ihren Alltag zu integrieren, die Glukosemessung korrekt vorzunehmen, die erforderliche Insulindosis zu berechnen oder die Insulinwirkdauer zu beachten.

„Moderne Technologie hilft, die Therapie zu sichern und erleichtert das Diabetesmanagement für die Patienten“, so Kröger. Ein neues System, das im Mai 2011 die CE-Zertifizierung erhalten hat, ist FreeStyle InsuLinx. Für Diabetiker bestimmt, die schnell wirksames Insulin einsetzen, integriert das System die Blutzuckermessung und Insulindosisberechnung. Über einen Touchscreen ist das individuell einstellbare Gerät leicht und intuitiv zu bedienen, die integrierte PC- und Mac-kompatible Software ermöglicht es Patienten und medizinischem Fachpersonal, Berichte $\mathrm{zu}$ dokumentieren sowie Erinnerungen und Meldungen zu verwalten. Der Hersteller stellt das Gerät kostenlos zur Verfügung.

koc

1. Whitmer RA et al., JAMA 2009; 301(15): 1565-72.

Quelle: Einführungsveranstaltung FreeStyle InsuLinx, 3.5.2011 in Frankfurt am Main, Veranstalter: Abbott

Kurz notiert

\section{Gliptin besonders effektiv und sicher - auch bei Über-75-Jährigen}

Obwohl die Gliptine einer Wirkstoffklasse angehören und am selben Enzym ansetzen, sind sie doch verschiedene Moleküle mit unterschiedlichen Eigenschaften. Vergleicht man die beiden Präparate, die zuerst erhältlich waren, Sita- und Vildagliptin, ist ihr Bindungverhalten an die DPP-4 nicht gleich: Sitagliptin ist ein kompetitiver Antagonist, bindet also an das Zielenzym und blockiert es, ohne einen Effekt auszulösen. Dabei konkurriert es mit dem Substrat GLP-1. Dies hat zur Folge, dass es relativ leicht von seiner Bindung verdrängt werden kann, wenn der GLP-1-Spiegel hoch ist. Vildagliptin (Galvus ${ }^{\oplus}$, plus Metformin Eucreas ${ }^{\circledast}$ ) aber wird von der DPP-4 metabolisiert, was eine feste kovalente Bindung von etwa einer Stunde bedingt, unabhängig vom GLP-1Spiegel. Als Hinweis auf dieses stärkere Wirkpotenzial können die Ergebnisse einer Studie von Marfalla et al. gewertet werden [1]. Darin hatte Vildagliptin zu postprandial höheren GLP-1-Plasma-Spiegeln geführt als Sitagliptin. Eine Besonderheit bei Vildagliptin ist zudem, dass es nicht in darstellbarem
Maß vom Enzymkomplex Cytochrom P 450 der Leber verstoffwechselt wird, hier also auch weder induziert noch hemmt. Dies ist in Anbetracht der Polypharmazie vieler Diabetiker wichtig. Zusätzlich ist der Einsatz von Vildagliptin bei Typ-2-Diabetikern $\geq 75$ Jahren ohne Dosisanpassung und ebenso sicher und effektiv möglich wie bei Jüngeren [2].

spa

1. Marfalla R et al. J Diabetes Compl 2010;24:79-83.

2. Schweizer et al. Diabetes, Obes Metab 2011,13:55-64. Quelle: Novartis

\section{Spezialschuh erhält Hilfsmittel-Nr.}

Nach dem Spezialschuh für Diabetiker LucRo classic hat es nun auch der LucRo kinetic als zweite Modellreihe des Unternehmens Schein in das Hilfsmittelverzeichnis geschafft. Der im Rückfuß schmalere kinetic ist nun unter der Hilfsmittelnummer 31.03.08.0008 erhältlich, sodass sich die Optionen zur Verordnung vergrößert haben. Beide Kollektionen zählen zu den wenigen Diabetiker-Spezialschuhen, die Hilfsmittelnummern haben und sich verschreiben lassen. red Infos unter www.lucro.de Quelle: Schein 